\title{
A review of molecular imaging of atherosclerosis and the potential application of dendrimer in imaging of plaque
}

This article was published in the following Dove Press journal:

International Journal of Nanomedicine

19 October 2017

Number of times this article has been viewed

\author{
Gulinigaer Anwaier ${ }^{1-3, *}$ \\ Cong Chen ${ }^{1,2, *}$ \\ Yini Cao ${ }^{1,2}$ \\ Rong $\mathrm{Qi}^{1-3}$ \\ 'Peking University Institute \\ of Cardiovascular Sciences, \\ Key Laboratory of Molecular \\ Cardiovascular Sciences, Ministry \\ of education, Peking University \\ Health Science Center, ${ }^{2}$ Beijing \\ Key Laboratory of Molecular \\ Pharmaceutics and New Drug \\ Delivery Systems, Beijing, ${ }^{3}$ School \\ of Basic Medical Science, Shihezi \\ University, Shihezi, Xinjiang, People's \\ Republic of China \\ *These authors contributed equally \\ to this work
}

\begin{abstract}
Despite the fact that technological advancements have been made in diagnosis and treatment, cardiovascular diseases (CVDs) remain the leading cause of mortality and morbidity worldwide. Early detection of atherosclerosis (AS), especially vulnerable plaques, plays a crucial role in the prevention of acute coronary syndrome (ACS). Targeting the critical cytokines and molecules that are upregulated during the biological process of AS by in vivo molecular imaging has been widely used in plaque imaging. With their three-dimensional architecture, composition, and abundant terminal functional groups, dendrimers provide a platform for multitargeting and multimodal imaging. Thus, modified dendrimers with the key molecules upregulated in AS plaques will be an innovative attempt to achieve targeted imaging of AS plaques specifically and efficiently. This review was aimed to address some recent works on imaging of AS plaques using various types of image technology and further discuss the applications of dendrimers, an innovative yet seldom used method in imaging of AS plaques due to some limitations and challenges, and we highlight the bright future of the modified dendrimers in characterizing AS plaques.
\end{abstract}

Keywords: atherosclerosis plaques, molecular imaging, dendrimers

\section{Introduction}

Despite the continuous and significant advances that have been made in diagnosis and therapies over the past decades, cardiovascular diseases (CVDs), including unstable angina, stroke, acute myocardial infarction with or without ST elevation, and sudden coronary death, remain the principal cause of mortality and morbidity globally. ${ }^{1}$

Most of the acute coronary syndromes (ACSs) are believed to be triggered by atheromatous plaque rupture. ${ }^{2,3}$ A vulnerable plaque contains a large lipid core and a thin fibrous cap with severe inflammatory cell infiltration, causing the release of matrix metalloproteinase (MMP). Released MMPs further weaken the fibrous cap, thus leading to disruption of the plaque cap, after which contents of the necrotic core, especially the collagen fibers, are exposed to the vessel lumen, resulting in thrombosis, which has been recognized as the pathological basis of many cardiovascular events. Therefore, advanced methods for early prevention as well as treatment of atherosclerosis (AS) are critically needed. ${ }^{4}$

At present, commonly used imaging techniques, ${ }^{5}$ including ultrasound (US), radiography, computed tomography (CT), nuclear medicine, eg, positron emission tomography $(\mathrm{PET})^{6-8}$ and single photon emission CT (SPECT), ${ }^{9,10}$ and magnetic resonance imaging (MRI). ${ }^{11}$ And MRI includes T1-weighted (T1w) and T2-weighted 
Table I Commonly used imaging techniques for AS diagnosis

\begin{tabular}{ll}
\hline Types & Principles of imaging \\
\hline US & Ultrasound is a kind of sound wave with a frequency greater than the upper limit of human hearing \\
$(>22 \mathrm{kHz})$ and ultrasound devices operate with frequencies from $20 \mathrm{kHz}$ up to several gigahertz \\
to detect objects and measure distances \\
Nuclear medicine records radiation emitting from within the body, and for such reason, it is called \\
a physiological imaging modality \\
The system detects pairs of gamma rays emitted indirectly by a positron-emitting radionuclide \\
(tracer), which has been introduced into the body on a biologically active molecule \\
PET & $\begin{array}{l}\text { It is similar to conventional nuclear medicine planar imaging using a gamma camera. However, it is } \\
\text { able to provide true 3D information }\end{array}$ \\
SPECT & $\begin{array}{l}\text { It uses electromagnetic radiation other than visible light, especially X-rays, to view the internal } \\
\text { Structure of a nonuniformly composed and opaque object } \\
\text { Radiography }\end{array}$ \\
A CT scan use computer-processed combinations of many X-ray images taken from various angles \\
to produce cross-sectional (tomographic) images (virtual "slices") of specific areas of a scanned \\
object, allowing the user to see inside the object without cutting \\
MRI scanners use strong magnetic fields, radio waves, and field gradients to generate images of the \\
inside of the body. And, it includes TI/T2-weighted MR
\end{tabular}

Abbreviations: AS, atherosclerosis; CT, computed tomography; 3D, three-dimensional; MR, magnetic resonance; MRI, magnetic resonance imaging; PET, positron emission tomography; SPECT, single photon emission CT; US, ultrasound.

(T2w) MRI ${ }^{12-14}$ or magnetic resonance angiography (MRA) scans ${ }^{15}$ and also many other optical imaging methods, all play a key role in the diagnosis of AS (Table 1). ${ }^{16,17}$

The development of medical imaging technology underwent three revolutionary innovation processes from structure imaging to functional imaging and to molecular imaging. Traditional intravascular invasive imaging techniques, including intravascular ultrasound (IVUS), acoustic contrast (ultrasonic contrast), intravascular MRI (IVMRI), and optical coherent tomography (OCT), are put emphasis mainly on the observation of internal structure of the vessel; however, the invasive procedures are costly and dangerous in terms of certain complications, such as wound infection, thrombosis formation, fragment detachment, and even artery rupture. ${ }^{18-21}$ Nowadays, with the development of imaging and advances in molecular biology, noninvasive molecular imaging becomes more and more important in disease diagnosis. Molecular imaging is a new trend in recent years, which combines molecular biology and in vivo imaging together to make cellular functions visualized, and traces molecular process in vivo as a noninvasive evaluating method. ${ }^{20}$ Usually, disease-related biomarkers, which are upregulated during the pathological process of the diseases, are needed in molecular imaging to target specific cells or molecules.,22-24 For AS diagnosis, molecular imaging with target-specific probes has shown great promise for noninvasive in vivo monitoring of biological processes of atheromatous plaques at molecular and cellular levels in both animals and humans. By targeting the specific molecules, the contrast agents can be located to atherosclerotic lesions accurately and signal intensity of different imaging techniques can thus be increased.

Dendrimers are a somewhat highly branched, monodispersed, and artificially synthetic macromolecules. Their unique well-defined three-dimensional architecture, composition, and abundant terminal functional groups make them potential carriers for imaging agents and drugs (Figure 1). ${ }^{25}$ For imaging, dendrimers can be modified by two or more molecules or imaging agents and have been applied for targeted imaging of many diseases such as cancer for many years. ${ }^{26}$ For example, some researchers have been studying dendrimers labeled with gold $(\mathrm{Au})$ or gadolinium $(\mathrm{Gd})$ in imaging of AS plaques using CT or magnetic resonance (MR), which would allow researchers or surgeons to locate nidi accurately and therefore treat the diseases efficiently. Here, we give a review on the present progress of AS plaque imaging and further discuss the applications and prospects of dendrimers in characterization of the AS plaques.

\section{Pathophysiology of AS and biomarkers for molecular target imaging}

Molecular imaging provides great potential for noninvasive visualization of the cellular and molecular components involved in the development of atheromatous plaques 

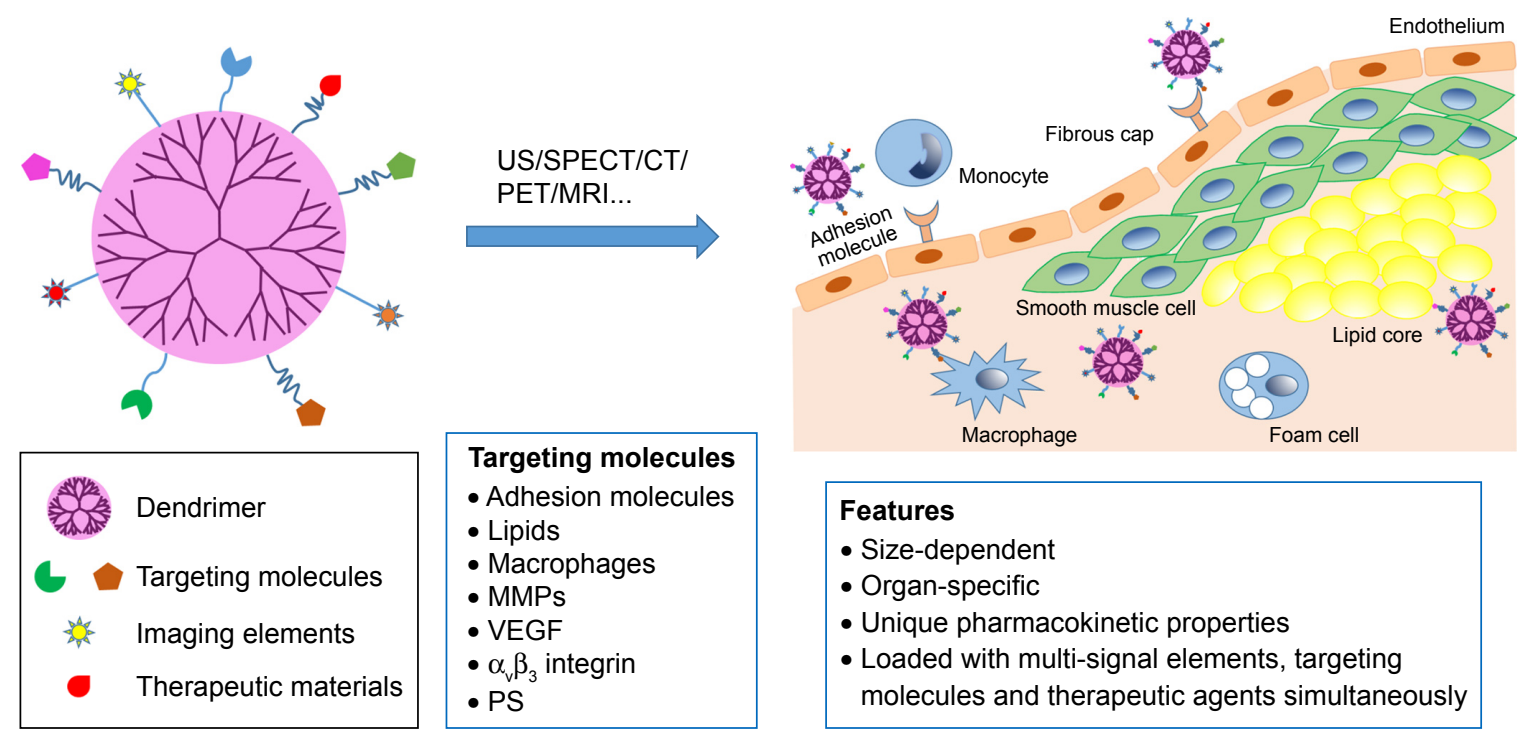

Features

- Size-dependent

- Organ-specific

- Unique pharmacokinetic properties

- Loaded with multi-signal elements, targeting

molecules and therapeutic agents simultaneously

Figure I Dendrimer-facilitated molecular imaging of atherosclerosis plaque.

Note: Dendrimer modified with multi-signal elements or therapeutic agents can target at a series of molecules that are upregulated in plaque area during AS, and can function as contrast agents or therapeutic agents there.

Abbreviations: AS, atherosclerosis; US, ultrasound; CT, computed tomography; SPECT, single photon emission CT; PET, positron emission tomography; MRI, magnetic resonance imaging; MMP, matrix metalloproteinase; VEGF, vascular endothelial growth factor; PS, phosphatidylserine.

(Table 2). We first discuss some key molecular imaging modalities and approaches for noninvasive detection of biological processes of atherogenesis, including inflammatory infiltration (described as upregulation of endothelial adhesion molecules and infiltration of macrophages), fibrotic responses, and eventually the formation of vulnerable plaques.

\section{The formation of plaques}

In the early stage of atherogenesis, hypercholesterolemia accounts for the vascular endothelial dysfunction, which is characterized by induced endothelial activation and increased endothelial permeability, arterial infiltration of leukocyte, platelet activation, and proliferation of smooth muscle cells. ${ }^{27}$ The increased endothelial permeability allows lipids to penetrate across the arterial wall, especially oxidized low-density lipoprotein (oxLDL), which can impair endothelial cell (EC) and induce expression of growth factors by the damaged EC, including monocyte chemotactic protein-1 (MCP-1), platelet-derived growth factor (PDGF), transforming growth factor beta (TGF- $\beta$ ), vascular cell adhesion molecule-1 (VCAM-1), intercellular adhesion molecule-1 (ICAM-1), and P-selectin and E-selectin expressed on the activated endothelial surface. This in turn promotes recruitment of monocytes to the vessel wall where monocytes roll along the activated endothelium and continuously absorb oxLDL through surface receptors, finally forming foam cells. These molecules further impair the function of EC. Therefore, endothelial dysfunction and lipid accumulation can be imaging biomarkers for early detection of AS. ${ }^{1}$
Some researchers have developed certain peptides that can target VCAM-1, which has shown high affinity and specificity for characterizing VCAM-1 both in vitro and in vivo. ${ }^{28-30}$ Jansen et al imaged lipids in atheromatous plaques using spectroscopic intravascular photoacoustics (sIVPA) to understand the progress of AS. ${ }^{31}$

Low-density lipoprotein (LDL) oxidation is a vital step for the initiation of atherosclerotic plaque formation, and a variety of oxidation-specific epitopes (OSEs) can generate by oxLDL. OSEs, which are not only expressed on oxLDL but also appeared on apoptotic cells and proteins in the extracellular matrix of atherosclerotic vessel wall, are proved immunogenic, proinflammatory, and proatherogenic. OSEs are usually recognized by innate pattern recognition receptors (PRRs), including scavenger receptors (SRs), which are generally present on the surface of dendritic cells and monocyte/macrophages, and also by some innate proteins, and these receptors or proteins further activate the innate immune responses, which normally are protective but can be proatherogenic if overactivated. ${ }^{32-34}$ Thus, OSEs have been developed as targets of noninvasive imaging of AS plaques. Briley-Saebo et $\mathrm{l}^{35}$ generated manganese (Mn(II)) molecular imaging probes targeting to OSEs with oxidation-specific antibodies MDA2 and IK17. After administration of the Mn-micelles for 48-72 h, strong MR signal enhancement was observed, with localization of the Mn-micelles within intraplaque macrophages. The results demonstrated that OSEs have the potential to be biomarkers of AS plaques and could be used in its molecular imaging in the future. 
Table 2 AS biomarkers used in molecular imaging

\begin{tabular}{|c|c|c|c|c|}
\hline Biomarkers & Molecular imaging probes & $\begin{array}{l}\text { Imaging } \\
\text { technique }\end{array}$ & Author & References \\
\hline VCAM-I & $\begin{array}{l}\text { Validate an (18)F-labeled small VCAM-I affinity ligand and } \\
\text { demonstrate the feasibility of imaging VCAM-I expression by PET-CT }\end{array}$ & $\begin{array}{l}\text { PET-CT } \\
\text { MRI }\end{array}$ & $\begin{array}{l}\text { Nahrendorf } \\
\text { et al }\end{array}$ & $\mathrm{II}, 28-30,114$ \\
\hline Lipids & $\begin{array}{l}\text { sIVPA imaging identifies lipids in human coronary AS plaque by relying } \\
\text { on contrast in the near-infrared absorption spectra of the arterial wall } \\
\text { components }\end{array}$ & sIVPA & Jansen et al & 31 \\
\hline OSEs & $\begin{array}{l}\text { Manganese }(\mathrm{Mn}(\mathrm{II})) \text { molecular imaging probes targeting to OSEs with } \\
\text { oxidation-specific antibodies MDA2 and IKI7 }\end{array}$ & MR & $\begin{array}{l}\text { Briley-Saebo } \\
\text { et al }\end{array}$ & 35 \\
\hline $\begin{array}{l}\text { Macrophages } \\
\text { CD204 }\end{array}$ & Using anti-CD204 monoclonal lgG & MRI & $\begin{array}{l}\text { Amirbekian } \\
\text { et al }\end{array}$ & $40-44$ \\
\hline \multirow[t]{2}{*}{ LOX-I } & $\begin{array}{l}\text { Imaging probe consisting of liposomes loaded with LOX-I antibody or } \\
\text { nonspecific immunoglobulin G }\end{array}$ & $\begin{array}{l}\mathrm{SPECT} / \mathrm{CT} \text { and } \\
\mathrm{MR}\end{array}$ & Li et al & 47 \\
\hline & $\begin{array}{l}\text { LOX-I antibody conjugated to USPIO nanoparticles could take the } \\
\text { probe to vulnerable plaques, which will be verified by colocalization } \\
\text { with vulnerable plaque biomarker }\end{array}$ & MRI & Wen et al & 50 \\
\hline Stable plaque & $\begin{array}{l}\text { Using the novel CD80-specific probe discriminated the stable ones } \\
\text { from vulnerable atherosclerotic plaques }\end{array}$ & PET & Muller et al & 51 \\
\hline \multirow[t]{3}{*}{ MMP } & Synthesized radiolabeled MPIs & $\begin{array}{l}\text { Radionuclide } \\
\text { imaging }\end{array}$ & Hartung et al & 58 \\
\hline & $\begin{array}{l}\text { A kind of MPI labeled with }{ }^{99 \mathrm{~m}} \mathrm{Tc} \text { could be used as an MMP-targeted } \\
\text { tracer }\end{array}$ & Micro-SPECT & $\begin{array}{l}\text { Razavian M } \\
\text { et al }\end{array}$ & 59 \\
\hline & $\begin{array}{l}\text { Using broad-spectrum MMP inhibitor HO-CGS } 27023 \mathrm{~A} \text {, labeled with } \\
{ }^{123} \text { or }{ }^{125} \text { image MMP activity }\end{array}$ & Scintigraphy & Schafers et al & 60 \\
\hline PS & $\begin{array}{l}\text { Utilizing Annexin A5 (like }{ }^{99 \mathrm{~m}} \mathrm{Tc} \text {-annexin A5) to target PS, which was } \\
\text { highly taken up in plaque rich in macrophages }\end{array}$ & SPECT/CT & Zhao et al & $68-70$ \\
\hline $\begin{array}{l}\text { Apoptosis (liposomes } \\
\text { contained PS) }\end{array}$ & Utility of paramagnetic Gd-PS & $\begin{array}{l}\text { Fluorescence plate } \\
\text { reader and MRI }\end{array}$ & $\begin{array}{l}\text { Maiseyeu } \\
\text { et al }\end{array}$ & 74 \\
\hline Caspase activity & $\begin{array}{l}\text { Utilizing a peptide-based caspase inhibitor for quantification of } \\
\text { caspase activity }\end{array}$ & PET & Hight et al & 75 \\
\hline Matrix remodeling & $\begin{array}{l}\text { Using an elastin-specific contrast agent to assess vascular remodeling } \\
\text { for the prediction of vulnerable plaques }\end{array}$ & MRI & $\begin{array}{l}\text { Phinikaridou } \\
\text { et al }\end{array}$ & 77 \\
\hline$\alpha_{v} \beta_{3}$ integrin $^{\mathrm{a}}$ & $\begin{array}{l}\text { Expression of } \alpha_{v} \beta_{3} \text { integrin by vasa vasorum was imaged at } 1.5 \mathrm{~T} \text { in } \\
\text { cholesterol-fed rabbit aortas using integrin-targeted paramagnetic } \\
\text { nanoparticles that incorporated fumagillin at } 0 \text { or } 30 \mu \mathrm{g} / \mathrm{kg}\end{array}$ & MRI & Winter et al & 76 \\
\hline $\mathrm{NGAL}^{\mathrm{a}}$ & $\begin{array}{l}\text { It is highly expressed in plaques and associated with increased } \\
\text { MMP-9 activity. Thus, enhancement of MR images on the abdominal } \\
\text { aorta of ApoE(-I-)/eNOS(-l-) mice and colocalization of micelles, } \\
\text { macrophages, and NGAL/24p3 were observed at } 72 \mathrm{~h} \text { after injection } \\
\text { of NGAL/24p3-targeted micelles }\end{array}$ & MRI & $\begin{array}{l}\text { te Boekhorst } \\
\text { et al }\end{array}$ & 52 \\
\hline VEGF & $\begin{array}{l}\text { Molecular near-infrared fluorescent imaging with an engineered } \\
\text { Cy5.5-labeled single-chain VEGF tracer (scVEGF/Cy) to detect VEGF } \\
\text { receptors and vascular remodeling in human coronary artery grafts by } \\
\text { molecular imaging }\end{array}$ & $\begin{array}{l}\text { Near-infrared } \\
\text { fluorescent } \\
\text { imaging }\end{array}$ & Zhang et al & 78 \\
\hline $\mathrm{CD} 40^{\mathrm{a}}$ & $\begin{array}{l}\text { A synthetic peptide corresponding with the phage insert NP3I } \\
\text { displayed nanomolar affinity for CD } 40 \text { and this design of a novel, } \\
\text { selective peptide ligand for CD } 40 \text { by phage display examined }\end{array}$ & $\begin{array}{l}\text { Truncation and } \\
\text { alanine scan } \\
\text { studies }\end{array}$ & Yu et al & 55 \\
\hline
\end{tabular}

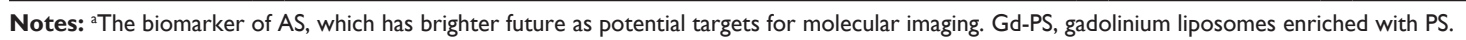

Abbreviations: AS, atherosclerosis; CT, computed tomography; LOX-I, Lectin-like oxidized low-density lipoprotein receptor-I; MMP, matrix metalloproteinase; MPIs, MMP inhibitors; MR, magnetic resonance; MRI, magnetic resonance imaging; NGAL, neutrophil gelatinase-associated lipocalin; OSEs, oxidation-specific epitopes; PET, positron emission tomography; PS, phosphatidylserine; sIVPA, spectroscopic intravascular photoacoustics; SPECT, single photon emission CT; USPIO, ultrasmall superparamagnetic iron oxide; VCAM-I, vascular cell adhesion molecule-I; VEGF, vascular endothelial growth factor.

\section{Inflammation (inflammatory infiltration)}

AS is a chronic inflammatory progression, which is regulated by innate and adaptive immune responses. ${ }^{36}$ Innate immunity usually based on the PRR on surface of macrophages and dendritic cells to identify various pathogens. PRRs are diverse, and SRs as well as Toll-like receptors (TLRs) are playing an important role in the formation of AS lesions. The initiation of adaptive immunity also can produce a large number of T cells, B cells, and immunoglobulin into AS lesion. Pathogenesis of AS lesions initially resulted from 
endothelial dysfunction, which then initiates T cells and macrophages to blood vessel walls and causes local infiltration of them, T cells then recognize oxLDL cholesterol, heat shock protein (HSP), and microbe antigen through molecular PRRs and induce the release of proinflammatory cytokines, causing complex interplay between different circulating factors and various cell types in the vessel wall. ${ }^{36-38}$ Then, it leads to the accumulation of lipids in the sub-endothelial space and a complex process of chronic inflammation, followed by migration of monocytes to endothelium and conversion of it to macrophages, which highly express a large number of SRs. ${ }^{39}$ When the lipids in the blood vessel are taken up by the macrophages through SRs and oxidized, the macrophages will change into foam cells with accumulation of esterified cholesterol. oxLDL can induce ECs to secrete MCP-1 as well as VCAM-1, which induce migration of macrophages to the lesion area. Macrophages in subcutaneous space will secrete various kinds of inflammatory cytokines that stimulate proliferation and migration of smooth muscle cells and proteolytic enzymes (eg, MMPs) that can degrade collagen and elastin, resulting in the thinning of the cap of the growing plaque and making the plaque susceptible to rupture. There is no doubt that the activated macrophages can be an important target for AS imaging, as they play such a key role in all stages of AS progression. Thus, targeting imaging of AS in many studies was also based on the accumulation of imaging particles in macrophages. ${ }^{40-44}$

Activated macrophages express some receptors and molecules on their surface, which can be targeted for plaque imaging. SRs such as SRA and SRB1, CD36, CD68, and TLRs $^{2}$ are significantly overexpressed on atherosclerotic macrophages and foam cells. Amirbekian et al ${ }^{40}$ used antiCD204 monoclonal IgG to target CD 204, a macrophage SR (MSR) for assessing AS in ApoE-/- mice by MRI, and found that macrophage-targeted immunomicelles significantly enhanced the uptake of imaging agent (by 79\%) compared to the untargeted immunomicelles (by 34\%). For the first time, it was discovered that MSR, a trimeric integral membrane glycoprotein that can bind to some proteins and molecules, could be detected due to its unusually broad ligand specificity and macrophages could be targeted in vivo with a positive contrast molecular construct designed for specific and sensitive assessment of AS. In addition, a large variety of polyanionic macromolecules, such as maleylated bovine serum albumin, have broad ligand specificity for several SRs overexpressed on the macrophages of the plaque and, therefore, can be applied as potential macrophagetargeting carriers. ${ }^{44}$
Lectin-like oxidized LDL receptor-1 (LOX-1, a kind of $\mathrm{SR}$ ) is an important receptor expressed on macrophages as well as some ECs and VSMCs under pathological conditions. oxLDL binding to LOX-1 will induce apoptosis as well as expression of adhesion molecules and MMP and activate the inflammatory pathway, all of which eventually contribute to the rupture of the plaques. ${ }^{45-48}$ In view of high expression of LOX on more than one kind of cells concerned in AS, LOX-1 will have great potential to be a target for molecular imaging of AS plaques. At present, imaging probes targeted at LOX-1 have been developed to improve sensitivity, specificity, and biocompatibility compared to the nontargeted ones. ${ }^{49,50}$ LOX-1 antibody-conjugated ultrasmall superparamagnetic iron oxide (USPIO) nanoparticles could be taken in vulnerable plaques in vivo, which could be verified by colocalization of the nanoparticles with vulnerable plaque biomarkers, such as macrophages and MMP-9. ${ }^{50}$ Li et $\mathrm{al}^{47}$ prepared an imaging probe consisting of liposomes loaded with LOX-1 antibody or nonspecific immunoglobulin $\mathrm{G}$ and tested their imaging effects using SPECT/CT and magnetic resonance. The LOX-1 targeted probes could bind to atherosclerotic plaques, especially to the shoulder areas of the plaque, a region with features of vulnerable plaque and LOX-1 expresses extensively, while nontargeted ones could not. The results were confirmed by observing no signal enhancement in $\mathrm{LDLR}^{-/-} / \mathrm{LOX}_{-1}^{-/-}$mice.

Moreover, inflammatory response is a complex process with many cytokines and receptors expressed in the progression of AS. Using a novel CD80-specific probe, Muller et $\mathrm{al}^{51}$ discriminated the stable atherosclerotic plaques from the vulnerable ones in vitro. Neutrophil gelatinase-associated lipocalin (NGAL), which is highly expressed in plaques and associated with increased MMP-9 activity, may be developed as a new imaging target for the diagnosis of high-risk plaques. ${ }^{52}$ In addition, chemokine receptors as well as IL-10 and CD40 can serve as targets for noninvasive imaging of atherosclerotic plaques. ${ }^{53-55}$

\section{Fibroatheromas}

Fibroatheromas consist of a necrotic core and a thick fibrous cap. The necrotic core is a result of macrophage infiltration with the release of MMP and macrophage apoptosis. The thick fibrous cap is composed of collagen, proteoglycans, and smooth muscle cells. The fibrous cap can be thinned by MMPs as well as apoptotic smooth muscle cells, leading to the rupture of the plaque cap. ${ }^{2}$

MMPs are a complex family of zinc-dependent endopeptidases that are usually characterized as secreted 
proteases responsible for degrading extracellular matrix components. ${ }^{56,57}$ MMP activity in the vessel wall is based on its expression level, activation state, and presence of its inhibitors. ${ }^{3}$ Overexpression of MMPs is a main factor in the transformation of plaques from stable to vulnerable. Thus, MMP-targeted imaging may have bright future in diagnosing vulnerable plaques. To characterize MMPs in plaque, some radiolabeled MMP inhibitors (MPIs) have been synthesized and employed. High radio signal could be detected in the segment with higher MMP expression for MPI targeting. ${ }^{58}$ RP805, a kind of MPI labeled with ${ }^{99 \mathrm{~m}} \mathrm{Tc}$, could be used as an MMP-targeted tracer for microSPECT imaging. ${ }^{59}$ Schafers et a ${ }^{60}$ used another broad-spectrum MMP inhibitor HO-CGS $27023 \mathrm{~A}$, labeled with ${ }^{123} \mathrm{I}$ or ${ }^{125} \mathrm{I}$, to image MMP activity in vivo in ApoE-/- mice using scintigraphy. Compared to the blockade group, there was $\sim 1.5$-fold increase in accumulation of the imaging agent caused by the specific uptake of the inhibitor HO-CGS 27023A in the plaque. The molecular imaging of MMP activity in lesions of AS may help predict and prevent against coronary events caused by plaque rupture.

\section{The formation of vulnerable plaques}

With the release of MMPs and the apoptosis of SMCs, the fibroatheromas transform into vulnerable plaques, which have a larger necrotic core and a thinner fibrous cap. ${ }^{3,61}$ Other features of vulnerable plaques include multiplication of inflammatory cells, especially in the fibrous cap, and large number of apoptotic cells (originally in the form of monocytes/macrophages and VSMCs). The infiltration of the inflammatory cells causes compensatory angiogenesis to resist hypoxia circumstances. However, neovessels will further enhance infiltration of inflammatory cells, leading to the release of proteolytic enzymes, causing excessive extracellular matrix degradation, which in turn induces matrix remodeling. ${ }^{3}$ Moreover, fragile neovessels are prone to rupture, leading to intraplaque hemorrhage ${ }^{62,63}$ Furthermore, all the pathological process of AS mentioned earlier including hemodynamics, endothelial dysfunction, and inflammation would lead to vascular remodeling, ${ }^{64}$ which usually involved in cell growth, apoptosis, migration, and extracellular matrix synthesis, degradation as well as rearrangement, to increase plaque vulnerability. ${ }^{65}$ While plaque formation or intimal hyperplasia has been recognized as a major determinant of AS luminal stenosis for many years, increasing numbers of studies on AS showed that vascular remodeling may be the another key factor of narrowing lumen size, aggravating the formation and rupture of unstable plaque. ${ }^{66,67}$ Therefore, all these characteristics possessed by the vulnerable plaques are potential targets for molecular imaging.

Phosphatidylserine (PS), a protein usually expressed on the outer surface of apoptotic cell membranes, also contributes to the rupture of vulnerable plaques. Annexin A5 is a protein, which possesses a nanomolar binding affinity to PS. Thus, researchers have utilized Annexin A5 to target PS for noninvasive imaging of atherosclerotic plaques. ${ }^{68-70}$ For example, ${ }^{99 \mathrm{~m}} \mathrm{Tc}$-annexin A5 was injected to animals for noninvasive in vivo SPECT/CT imaging and the lesions were clearly visualized. The ${ }^{99 \mathrm{~m}} \mathrm{Tc}$-annexin A5 was highly taken up in the plaques rich in macrophages. Through histological and immunohistochemical evaluations, a significant correlation between radiotracer uptake and both macrophage infiltration and the extent of apoptosis was revealed. ${ }^{71}$ As is well known to all, exteriorized PS residues expressed on apoptosis cells accelerate their recognition by macrophages via CD36, which triggers rapid phagocytosis. ${ }^{72-74}$ Maiseyeu et al ${ }^{74}$ mimicked apoptosis using liposomes containing PS and looked into the utility of paramagnetic Gd liposomes enriched with PS (Gd-PS) using fluorescence plate reader as well as MRI scanner in imagining AS plaques. They found rapid and significant enhancement of the aortic wall both in vivo and in vitro, thus such Gd-PS liposomes can provide a new targeting approach of contrast agent for imaging macrophage. In addition, caspase is a promising target for imaging apoptosis. Hight et $\mathrm{al}^{75}$ have successfully utilized a peptide-based caspase inhibitor for the quantification of caspase activity by PET.

$\alpha_{v} \beta_{3}$ integrin can be targeted to image neovascularization, as it is only expressed by ECs of neovascellum, proliferating VSMCs and activated macrophages. ${ }^{76}$ For imaging matrix remodeling, Phinikaridou et al ${ }^{77}$ successfully assessed vascular remodeling for the prediction of vulnerable plaques with an elastin-specific contrast agent. In addition, vascular endothelial growth factor (VEGF) plays a key role in vascular remodeling. Imaging methods targeted at VEGF receptor have a promising future for detecting vascular remodeling. ${ }^{78}$

\section{Limitations for current contrast agents}

Although plenty of approaches have been evaluated to image AS plaques and showed great prospects for further basic and clinical studies, there remain certain limitations and challenges for plaque-targeted imaging.

One of these limitations is the low sensitivity of some imaging modalities (MRI), which only target at a few biological molecules with high expression in AS plaques. ${ }^{14,79}$ For 
smaller coronary imaging, high temporal and spatial resolution are necessary, while for nonnuclear techniques, higher payloads of labeled molecules for imaging are required. Moreover, each imaging modality (CT, PET, or MR) has its particular strengths or weaknesses in terms of resolution, applicability to particular problems, and cost and requirement for equipment and external agents; thus, combination of different imaging techniques will be a trend for plaque imaging in the future. ${ }^{80}$

Besides, biological activity of targeted tracers also needs to be taken into considerations. Above all, substantial improvements have to be achieved for making contrast agents available. As we all known, the molecules overexpressed in the plaques vary in the different stage of AS progress. Although the previous studies have shown efficient plaque imaging by targeting one molecular as is mentioned earlier, single target imaging is hard to provide overall structural, functional, and molecular information of the targeted lesion area; therefore, multimodified target imaging agents will have great research value. Faced with these challenges, development of new tracers as contrast agents is of great significance, especially nanoparticles such as iron oxides, $\mathrm{Au}$, dendrimers, liposomes, micelles, and biodegradable polymers. ${ }^{17,81}$ Combination of specific targeting probes with imaging modalities for vulnerable plaque imaging is a research area of strong interest, especially for dendrimers. ${ }^{82,83}$

\section{Dendrimers as imaging agents}

In in vivo, the degradation and excretion of macromolecules are slower than those of small molecules, which resulted in increased relaxivity (remained in plasma for at least $1 \mathrm{~h}$ ), lengthened circulation clearance, and prolonged retention in blood pool. At the same time, due to the slow rotation of the molecular volume ambassador, the rotation correlation time is prolonged and the relaxation rate of the water quality can be remarkably increased. Therefore, the use of macromolecular contrast agents can reduce the dosage and enhance the examination of multiple parts of the body. ${ }^{84}$ Since early 1980s, dendrimers have been comprehensively studied for their various medical applications. ${ }^{85,86}$ Typically, a dendrimer molecule is composed of the following three elements: an initiator core, interior layers, which are attached to the initiator core and are built of repeating units (number of the layers are expressed as generations), and multiple terminal functional groups. ${ }^{87}$ Due to their unique characteristics, utilization of dendrimers in nanobiotechnology, such as bio-mimicry, diagnostics, and therapeutics, has attracted great attention of researchers over the past decade.
Previous studies have revealed many advantages of dendrimers in molecular imaging (Table 3).

First, dendrimer-based imaging agents exhibit sizedependent, organ-specific, and unique pharmacokinetic properties due to the characteristics of dendrimers, such as nanospherical size, cores, and interiors, as well as chemical properties of exterior shell, which are superior to other nanoparticles. Compared to low-molecular weight vehicle, which is likely to be degraded and excreted, polyamidoamine (PAMAM) dendrimers are easy to "leak" across vascular wall causing rapid perfusion throughout the body. ${ }^{79,88-90}$

Second, as macromolecular imaging agents, dendrimers, especially those modified with polyethylene glycol (PEG), can overcome some disadvantages of the traditional small molecular imaging agents, such as short half-life, nonspecificity, and renal toxicity at high concentrations. The potential toxicity of dendrimers can also be reduced by changing the types of central core or modified with anionic, neutral, and ligand molecules. ${ }^{17,25,79,91}$

In addition, dendrimers can be labeled with a variety of imaging "beacons" and such combination of dendrimers and beacons can be used as hybrid contrast agent probe, which lengthens circulation clearance and prolongs retention in blood pool but can be cleared away from major organs in short time in different imaging methods. ${ }^{25,79,90,92}$

More importantly, multiple surface functional groups of dendrimers can be labeled with multisignal elements, targeting molecules and therapeutic agents. ${ }^{92,93}$ Therefore, dendrimers can provide a wide range of space and binding sites for functional molecules and elements, which enables them to have more abilities and advantages for targeted molecular imaging than other nanoparticles.

Up to now, more and more researchers have been concentrating on the utilization of dendrimers as imaging agents. In the early days, dendrimers were only used as vectors of some imaging elements; for example, Mn(II) or Gd(III) chelating dendrimers were prepared as contrast agents for MRI applications ${ }^{88,91,94}$ dye-conjugated dendrimers were used for fluorescence imaging; ${ }^{95,96}$ and dendrimer-entrapped $\mathrm{Au}$ nanoparticles (Au DENPs) or iodinated-compounds conjugated dendrimers were applied to CT. ${ }^{97-100}$

With multi-signal elements and targeting molecules in one dendrimer molecule, dendrimers can easily target to the intended imagining organs. Dendrimers conjugated with folic acid (FA) and $\mathrm{Cu}^{2+}$ were studied as bioprobes to target and image human cancers using synchrotron X-ray fluorescence analysis, and the results showed that the targeting probes increased the intensity of fluorescence signal compared to 
Table 3 Dendrimers based molecular imaging agents

\begin{tabular}{|c|c|c|c|c|}
\hline Contrast agents & Disease & Modality & Author & References \\
\hline $\mathrm{Mn}(\mathrm{II})$ or $\mathrm{Gd}(\mathrm{III})$ chelating dendrimers & Soft tissues & MRI & Klemm et al & $88,91,94$ \\
\hline Dye-conjugated dendrimers & $\begin{array}{l}\text { Single proteins and } \\
\text { biomolecules in cells }\end{array}$ & $\begin{array}{l}\text { Fluorescence } \\
\text { imaging }\end{array}$ & Kim et al & 95,96 \\
\hline Dendrimer-entrapped AuNPs & Blood pool and tumor & $\mathrm{CT}$ & Peng et al & $97-100$ \\
\hline Dendrimers conjugated to iodinated-compounds & $\begin{array}{l}\text { Human hepatocellular } \\
\text { carcinoma }\end{array}$ & $\mathrm{CT}$ & Liu et al & $97-100$ \\
\hline Dendrimers conjugated with $\mathrm{FA}$ and $\mathrm{Cu}^{2+}$ & Cancers & $\begin{array}{l}\text { Synchrotron X-ray } \\
\text { fluorescence analysis }\end{array}$ & Zhang et al & 93 \\
\hline Folate-receptor targeted dendrimer nanoclusters labeled with Gd & Cancers & MR & Cheng et al & 26 \\
\hline $\begin{array}{l}\text { Dendrimer-based contrast agents modified on an atom-by- } \\
\text { atom scale }\end{array}$ & $\begin{array}{l}\text { Organs and other } \\
\text { target-specific locations }\end{array}$ & $\begin{array}{l}\text { Hybrid modality } \\
\text { imaging }\end{array}$ & Longmire et al & 79 \\
\hline $\begin{array}{l}\text { Au DENPs loaded with Gd chelator/Gd(III) complexes modified } \\
\text { with RGD peptide }\end{array}$ & Tumors & $\mathrm{CT}$ and $\mathrm{MR}$ & Chen et al & 101,102 \\
\hline Gd-loaded dendrimer-entrapped AuNPs & $\begin{array}{l}\text { Major organs of rats } \\
\text { or mice }\end{array}$ & CT/MR & Wen et al & 25 \\
\hline $\begin{array}{l}\text { Dendrimer modified with AuNP followed by Gd(III) chelation } \\
\text { and acetylation of the remaining dendrimer terminal amine } \\
\text { groups (Gd-Au DENPs) }\end{array}$ & Breast cancer & CT/MR & Li et al & 103 \\
\hline FA-modified multifunctional Au DENPs loaded with Gd & Tumors & $\mathrm{CT} / \mathrm{MR}$ & Chen et al & 104 \\
\hline $\begin{array}{l}\text { A probe consisting of the fourth generation (G(4)-PAMAM) } \\
\text { dendrimer nanoparticles labeled with high-affinity conjugating } \\
\text { ligands and an organic fluorescent dye fluorescein }\end{array}$ & Mitochondria & CAT microscopy & Denora et al & 95 \\
\hline PEG modified Au DENPs ${ }^{a}$ & As plaques & $\mathrm{CT}$ & Ye et al & 107 \\
\hline Labeling Au DENPs with PEG and $\mathrm{Fl}^{\mathrm{a}}$ & AS & $\mathrm{CT}$ & Qin et al & 108 \\
\hline Dendrimers with ${ }^{64} \mathrm{Cu}$ modified with LyP-I ${ }^{a}$ & $\begin{array}{l}\text { p32 proteins on } \\
\text { activated macrophages }\end{array}$ & PET-CT & Seo et al & 109 \\
\hline G8 dendrimers modified by $M n$ and antibody $M{ }^{2} 2^{a}$ & As lesions & MRI & Nguyen et al & 110 \\
\hline
\end{tabular}

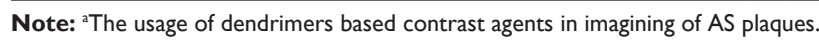

Abbreviations: AS, atherosclerosis; Au DENPs, dendrimer-entrapped gold nanoparticles; AuNP, gold nanoparticle; CAT, confocal-atomic force microscopy-total internal reflectance fluorescence; CT, computed tomography; FA, folic acid; Fl, fluorescein isothiocyanate; Gd, gadolinium; MR, magnetic resonance; MRI, magnetic resonance imaging; PEG, polyethylene glycol; RGD, arginylglycylaspartic acid; PET, positron emission tomography.

the negative control. ${ }^{93}$ Likewise, folate receptor-targeted dendrimer nanoclusters (DNCs) labeled with Gd can be used for MRI of cancers with high expression of folate-receptor. Cheng et $\mathrm{al}^{26}$ found that when KB cells, a cell line that can be induced to highly express folate receptor, were incubated with folate receptor-targeted DNCs, there was a significant enhancement in MR signal intensity compared to other cell lines with lower or normal expression of folate receptor.

In addition, dendrimers serve as vehicles for hybrid modality imaging due to their unique structures. ${ }^{79}$ It is known that Au nanoparticles are used for CT imaging while Gd(III) is for MRI. Combination of CT with MR was proved to possess advantages of both two imaging modalities. What have been confirmed in many researches is that dendrimers have superiority over other nanoparticles in loading contrast agents that have both CT and MRI elements. ${ }^{101,102}$ For instance, GD-loaded Au DENPs were synthetized for dual-modal CT/MRI applications. The new contrast agents possessed water solubility with stable colloid properties and were noncytotoxic within the given concentration range, which enabled efficient dual-modal CT/MRI of some major organs of rats and mice. ${ }^{25} \mathrm{Li}$ et al ${ }^{103}$ utilized the multifunctional dendrimer-based nanoparticles to image the breast cancer cells in vitro and in vivo by dual-modal CT/MR and proved effectiveness of the nanoparticles. Furthermore, Chen et al ${ }^{104}$ prepared FA-modified multifunctional Au DENPs loaded with Gd for target imaging of tumors by dual mode CT/MR and found that the probes showed both CT and MR enhancements in contrast with nontargeted Gd-Au DENPs. In addition, dendrimers have been applied for other hybrids such as dual-modal MR and fluorescence molecular imaging, MRI and other optical imaging, combination of molecular imaging and therapeutic agents, and multimodal contrast agents. ${ }^{79}$

And more notably, dendrimers have also been used as vehicles for both imaging agents and therapeutic materials. ${ }^{105}$ For example, Luong et al ${ }^{106}$ designed a theranostic nanocarrier consisting of superparamagnetic iron oxide nanoparticle (SPION) core and FA-PAMAM dendrimer surface. Further to increase its therapeutic potentials, an anticancer agent 3,4-difluorobenzylidene-curcumin (CDF) was loaded in 
the FA-PAMAM. The results showed that the SPIONs@ FA-PAMAM-CDF nanocarrier exhibited high MR contrast and a good anticancer activity simultaneously in vitro. This study indicates that multifunctions of targeting molecules for diagnosis and treatment can be combined within one molecule of dendrimers.

Most applications and attempts about dendrimers as contrast agents have focused on cancers, and researches about developing dendrimers for imaging of atherosclerotic plaques are very limited so far (Table 3 ). Ye et al ${ }^{107}$ used PEGmodified Au DENPs to image macrophages in atherosclerotic plaques using CT and proved that the PEGylated Au DENPs had great biocompatibility and were noncytotoxic even at high concentrations, and the Au DENPs were efficiently taken up by macrophages. These results were also confirmed by another study, in which Au DENPs were labeled with PEG and fluorescein isothiocyanate (FI). ${ }^{108}$ These achievements suggest that PEGylated Au DENPs could be an ideal contrast agent for CT imaging of AS.

About molecular target imaging, Seo et al ${ }^{109}$ modified dendrimers with LyP-1, which can bind to p32 proteins expressed on activated macrophages and labeled the dendrimers with ${ }^{64} \mathrm{Cu}$ for PET-CT studies. The (LyP-1) -dendrimer${ }^{64} \mathrm{Cu}$ was proved to be taken up in plaques significantly higher than nontargeted control, leading to the enhancement of signal intensity. Another element, Mn, is also suitable for imaging atherosclerotic plaques by the detection of OSEs in macrophage-rich atherosclerotic plaques using biocompatible $\mathrm{Mn}$ (II) molecular magnetic imaging probes. Mn can replace Gd to avoid potential cell toxicity of Gd, but high payloads of $\mathrm{Mn}$ are required for optimizing image technology. PAMAM dendrimers composed of repeating $\beta$-alanine subunits are able to load a large number of Mn. Nguyen et a $\mathrm{l}^{110}$ proved that G8 dendrimers modified by Mn and antibody MDA2 allowed a high Mn payload and targeted at OSE, which may have potentials in in vivo imaging of atherosclerotic lesions.

\section{Future perspectives}

Over the past few years, great efforts have been made to explore possible imaging agents to target different molecules overexpressed in AS plaques in order to achieve accurate diagnosis as well as efficiently deal with vulnerable plaques in primetime, but there lacks exciting progress.

At present, although applications of dendrimer for AS plaque imaging are still limited in quantity, dendrimers being studied as vehicles of contrast agents have shown great promise for molecular target imaging, dual- or multimodality imaging as well as targeted theranostics. If synthetic dendrimers are not only conjugated with one of the key molecules upregulated in AS plaques but also labeled by one or more imaging agents of diverse imaging modes, it will be very helpful for obtaining sufficient and accurate information about AS plaques. Furthermore, therapeutic agents can also be conjugated to dendrimer molecules to accomplish efficient treatment and diagnosis simultaneously.

Although they have extensive application value, dendrimers themselves still have some disadvantages to overcome. For example, the artificial synthesis of the high generations of dendrimers (G8-10) is expensive and complex, which make dendrimers have some difficulties in industrial production. Besides, due to their high molecular weights, the high generations of dendrimers generally have low diffusion coefficient and slow degradation and excretion, which may benefit for the dendrimers as contrast agents by prolonging retention of in blood pool, but also bring hemolysis and cellular toxicities simultaneously, ${ }^{84,111}$ especially for the cationic dendrimers because of their high density of positive charges. ${ }^{112,113}$

Therefore, optimized synthesis and purification methods will be encouraged to guarantee mass production of the dendrimers and modification of the dendrimers with PEG or anionic, neutral, and ligand molecules will not only effectively reduce their in vivo toxicities but also significantly increase their targeting capabilities, by which dendrimers would be applied widely as contrast agents in clinic for diagnosing and treating vulnerable plaques of AS in the near future.

\section{Executive summary Pathophysiology of AS and biomarkers for molecular target imaging}

- Molecular imaging provides great potential for noninvasive visualization of the cellular and molecular components involved in the development of atheromatous plaques.

- Some key molecular imaging modalities and approaches were used for noninvasive detection of biological processes of atherogenesis, including inflammatory infiltration (described as upregulation of endothelial adhesion molecules and infiltration of macrophages), fibrotic responses, and eventually the formation of vulnerable plaques.

\section{Limitations for current contrast agents}

- Each imaging modality (CT, PET, or MR) has its particular strengths or weaknesses in terms of resolution, applicability to particular problems, and cost and requirement 
for equipment and external agents; thus, combination of different imaging techniques will be a trend for plaque imaging in the future.

- Single target imaging is hard to provide overall structural, functional, and molecular information of the targeted lesion area; therefore, multi-modified target imaging agents will have great research value.

\section{Dendrimers as imaging agents}

- Due to their unique characteristics, utilization of dendrimers in nanobiotechnology, such as biomimicry, diagnostics, and therapeutics, has attracted great attention of researchers over the past decade.

- With their three-dimensional architecture, composition, and abundant terminal functional groups, dendrimers provide a platform for multitargeting and multimodal imaging.

- In addition, dendrimers have been used as vehicles for both imaging agents and therapeutic materials.

- Most applications and attempts about dendrimers as contrast agents have focused on cancers, and only a few attempts have focused on atherosclerotic plaques.

- Modified dendrimers are supposed to have great influence on imaging and treating vulnerable plaques, and it may be applied for clinical use in AS diagnosis and therapy in the future.

\section{Acknowledgment}

This work was supported by the National Natural Science Foundation of China (no 81270368).

\section{Disclosure}

The authors report no conflicts of interest in this work.

\section{References}

1. Phinikaridou A, Andia ME, Lacerda S, Lorrio S, Makowski MR, Botnar RM. Molecular MRI of atherosclerosis. Molecules. 2013; 18(11):14042-14069.

2. Sakakura K, Nakano M, Otsuka F, Ladich E, Kolodgie FD, Virmani R. Pathophysiology of atherosclerosis plaque progression. Heart Lung Circ. 2013;22(6):399-411.

3. Tavakoli S, Vashist A, Sadeghi MM. Molecular imaging of plaque vulnerability. J Nucl Cardiol. 2014;21(6):1112-1128. quiz 1129.

4. Karagkiozaki V, Logothetidis S, Pappa AM. Nanomedicine for atherosclerosis: molecular imaging and treatment. J Biomed Nanotechnol. 2015;11(2):191-210.

5. Adamson PD, Newby DE, Dweck MR. Translational coronary atherosclerosis imaging with PET. Cardiol Clin. 2016;34(1):179-186.

6. Catana C, Wu Y, Judenhofer MS, Qi J, Pichler BJ, Cherry SR. Simultaneous acquisition of multislice PET and MR images: initial results with a MR-compatible PET scanner. J Nucl Med. 2006;47(12): 1968-1976.

7. Ametamey SM, Honer M, Schubiger PA. Molecular imaging with PET. Chem Rev. 2008;108(5):1501-1516.
8. Mankoff DA, Lee JH, Eubank WB. Breast cancer imaging with novel PET tracers. PET Clin. 2009;4(4):371-380.

9. Ell PJ. Single photon emission computed tomography (SPET) of the brain. J Neurosci Methods. 1990;34(1-3):207-217.

10. Cheng D, Wang Y, Liu X, et al. Comparison of $18 \mathrm{~F}$ PET and $99 \mathrm{mTc}$ SPECT imaging in phantoms and in tumored mice. Bioconjug Chem. 2010;21(8):1565-1570.

11. Huang Y, Coman D, Hyder F, Ali MM. Dendrimer-based responsive MRI contrast agents (G1-G4) for biosensor imaging of redundant deviation in shifts (BIRDS). Bioconjug Chem. 2015;26(12):2315-2323.

12. Noguchi T, Kawasaki T, Tanaka A, et al. High-intensity signals in coronary plaques on noncontrast $\mathrm{T} 1$-weighted magnetic resonance imaging as a novel determinant of coronary events. J Am Coll Cardiol. 2014; 63(10):989-999.

13. Matsumoto K, Ehara S, Hasegawa T, et al. Localization of coronary high-intensity signals on T1-weighted MR imaging: relation to plaque morphology and clinical severity of angina pectoris. JACC Cardiovasc Imaging. 2015;8(10):1143-1152.

14. Xie Y, Kim YJ, Pang J, et al. Coronary atherosclerosis T1-weighed characterization with integrated anatomical reference: comparison with high-risk plaque features detected by invasive coronary imaging. JACC Cardiovasc Imaging. 2017;10(6):637-648.

15. Pang J, Sharif B, Arsanjani R, et al. Accelerated whole-heart coronary MRA using motion-corrected sensitivity encoding with threedimensional projection reconstruction. Magn Reson Med. 2015;73(1): 284-291.

16. Saam T, Hatsukami TS, Takaya N, et al. The vulnerable, or high-risk, atherosclerotic plaque: noninvasive MR imaging for characterization and assessment. Radiology. 2007;244(1):64-77.

17. Ryvolova M, Chomoucka J, Drbohlavova J, et al. Modern micro and nanoparticle-based imaging techniques. Sensors. 2012;12(11): 14792-14820.

18. Dash D. Optical coherence tomography is a kid on the block: I would choose intravascular ultrasound. Indian Heart J. 2017;69(3): 407-410.

19. Alsidawi S, Effat M, Rahman S, Abdallah M, Leesar M. The role of vascular imaging in guiding routine percutaneous coronary interventions: a meta-analysis of bare metal stent and drug-eluting stent trials. Cardiovasc Ther. 2015;33(6):360-366.

20. Herschman HR. Molecular imaging: looking at problems, seeing solutions. Science. 2003;302(5645):605-608.

21. Patel K, Tarkin J, Serruys PW, et al. Invasive or non-invasive imaging for detecting high-risk coronary lesions? Expert Rev Cardiovasc Ther. 2017;15(3):165-179.

22. Libby P, DiCarli M, Weissleder R. The vascular biology of atherosclerosis and imaging targets. $J$ Nucl Med. 2010;51(suppl 1):33S-37S.

23. Majmudar MD, Nahrendorf M. Cardiovascular molecular imaging: the road ahead. J Nucl Med. 2012;53(5):673-676.

24. Wang YX, Choi Y, Chen Z, Laurent S, Gibbs SL. Molecular imaging: from bench to clinic. Biomed Res Int. 2014;2014:357258.

25. Wen S, Li K, Cai H, et al. Multifunctional dendrimer-entrapped gold nanoparticles for dual mode CT/MR imaging applications. Biomaterials. 2013;34(5):1570-1580.

26. Cheng Z, Thorek DL, Tsourkas A. Gadolinium-conjugated dendrimer nanoclusters as a tumor-targeted T1 magnetic resonance imaging contrast agent. Angew Chem Int Ed Engl. 2010;49(2):346-350.

27. Profumo E, Buttari B, D'Arcangelo D, et al. The nutraceutical dehydrozingerone and its dimer counteract inflammation- and oxidative stress-induced dysfunction of in vitro cultured human endothelial cells: a novel perspective for the prevention and therapy of atherosclerosis. Oxid Med Cell Longev. 2016;2016:1246485.

28. Nahrendorf M, Keliher E, Panizzi P, et al. 18F-4V for PET-CT imaging of VCAM-1 expression in atherosclerosis. JACC Cardiovasc Imaging. 2009;2(10):1213-1222.

29. Michalska M, Machtoub L, Manthey HD, et al. Visualization of vascular inflammation in the atherosclerotic mouse by ultrasmall superparamagnetic iron oxide vascular cell adhesion molecule-1-specific nanoparticles. Arterioscler Thromb Vasc Biol. 2012;32(10):2350-2357. 
30. Dimastromatteo J, Broisat A, Perret $\mathrm{P}$, et al. In vivo molecular imaging of atherosclerotic lesions in ApoE-/- mice using VCAM-1specific, 99mTc-labeled peptidic sequences. J Nucl Med. 2013;54(8): 1442-1449.

31. Jansen K, van der Steen AF, Wu M, et al. Spectroscopic intravascular photoacoustic imaging of lipids in atherosclerosis. J Biomed Opt. 2014;19(2):026006.

32. Purushothaman KR, Purushothaman M, Levy AP, et al. Increased expression of oxidation-specific epitopes and apoptosis are associated with haptoglobin genotype: possible implications for plaque progression in human atherosclerosis. J Am Coll Cardiol. 2012;60(2):112-119.

33. van Dijk RA, Kolodgie F, Ravandi A, et al. Differential expression of oxidation-specific epitopes and apolipoprotein(a) in progressing and ruptured human coronary and carotid atherosclerotic lesions. J Lipid Res. 2012;53(12):2773-2790.

34. Leibundgut G, Witztum JL, Tsimikas S. Oxidation-specific epitopes and immunological responses: translational biotheranostic implications for atherosclerosis. Curr Opin Pharmacol. 2013;13(2):168-179.

35. Briley-Saebo KC, Nguyen TH, Saeboe AM, et al. In vivo detection of oxidation-specific epitopes in atherosclerotic lesions using biocompatible manganese molecular magnetic imaging probes. J Am Coll Cardiol. 2012;59(6):616-626.

36. Azzam KM, Fessler MB. Crosstalk between reverse cholesterol transport and innate immunity. Trends Endocrinol Metab. 2012;23(4): 169-178.

37. Ding YH, Qian LY, Pang J, et al. The regulation of immune cells by lactobacilli: a potential therapeutic target for anti-atherosclerosis therapy. Oncotarget. 2017;8:59915-59928.

38. Chistiakov DA, Orekhov AN, Bobryshev YV. Immune-inflammatory responses in atherosclerosis: role of an adaptive immunity mainly driven by T and B cells. Immunobiology. 2016;221(9):1014-1033.

39. Badimon L, Suades R, Fuentes E, Palomo I, Padro T. Role of plateletderived microvesicles as crosstalk mediators in atherothrombosis and future pharmacology targets: a link between inflammation, atherosclerosis, and thrombosis. Front Pharmacol. 2016;7:293.

40. Amirbekian V, Lipinski MJ, Briley-Saebo KC, et al. Detecting and assessing macrophages in vivo to evaluate atherosclerosis noninvasively using molecular MRI. Proc Natl Acad Sci U S A. 2007;104(3): 961-966.

41. Nahrendorf M, Zhang H, Hembrador S, et al. Nanoparticle PET-CT imaging of macrophages in inflammatory atherosclerosis. Circulation. 2008;117(3):379-387.

42. Nahrendorf M, Keliher E, Marinelli B, et al. Detection of macrophages in aortic aneurysms by nanoparticle positron emission tomographycomputed tomography. Arterioscler Thromb Vasc Biol. 2011;31(4): $750-757$.

43. Tarkin JM, Joshi FR, Rudd JH. PET imaging of inflammation in atherosclerosis. Nat Rev Cardiol. 2014;11(8):443-457.

44. Gustafsson B, Hedin U, Caidahl K. Glycolaldehyde and maleyl conjugated human serum albumin as potential macrophage-targeting carriers for molecular imaging purposes. Contrast Media Mol Imaging. 2015;10(1):37-42.

45. Dominguez JH, Mehta JL, Li D, et al. Anti-LOX-1 therapy in rats with diabetes and dyslipidemia: ablation of renal vascular and epithelial manifestations. Am J Physiol Renal Physiol. 2008;294(1): F110-F119.

46. Li D, Mehta JL. Intracellular signaling of LOX-1 in endothelial cell apoptosis. Circ Res. 2009;104(5):566-568.

47. Li D, Patel AR, Klibanov AL, et al. Molecular imaging of atherosclerotic plaques targeted to oxidized LDL receptor LOX-1 by SPECT/CT and magnetic resonance. Circ Cardiovasc Imaging. 2010;3(4):464-472.

48. Ding Z, Liu S, Wang X, et al. LOX-1, oxidant stress, mtDNA damage, autophagy, and immune response in atherosclerosis. Can J Physiol Pharmacol. 2014;92(7):524-530.

49. De Vos J, Mathijs I, Xavier C, et al. Specific targeting of atherosclerotic plaques in ApoE(-/-) mice using a new Camelid sdAb binding the vulnerable plaque marker LOX-1. Mol Imaging Biol. 2014;16(5): 690-698.
50. Wen S, Liu DF, Cui Y, et al. In vivo MRI detection of carotid atherosclerotic lesions and kidney inflammation in ApoE-deficient mice by using LOX-1 targeted iron nanoparticles. Nanomedicine. 2014; 10(3):639-649.

51. Muller A, Mu L, Meletta R, et al. Towards non-invasive imaging of vulnerable atherosclerotic plaques by targeting co-stimulatory molecules. Int J Cardiol. 2014;174(3):503-515.

52. te Boekhorst BC, Bovens SM, Hellings WE, et al. Molecular MRI of murine atherosclerotic plaque targeting NGAL: a protein associated with unstable human plaque characteristics. Cardiovasc Res. 2011; 89(3):680-688.

53. Almer G, Frascione D, Pali-Scholl I, et al. Interleukin-10: an antiinflammatory marker to target atherosclerotic lesions via PEGylated liposomes. Mol Pharm. 2013;10(1):175-186.

54. Liu Y, Pierce R, Luehmann HP, Sharp TL, Welch MJ. PET imaging of chemokine receptors in vascular injury-accelerated atherosclerosis. J Nucl Med. 2013;54(7):1135-1141.

55. Yu H, Segers F, Sliedregt-Bol K, et al. Identification of a novel CD40 ligand for targeted imaging of inflammatory plaques by phage display. FASEB J. 2013;27(10):4136-4146.

56. Back M, Ketelhuth DF, Agewall S. Matrix metalloproteinases in atherothrombosis. Prog Cardiovasc Dis. 2010;52(5):410-428.

57. Small CD, Crawford BD. Matrix metalloproteinases in neural development: a phylogenetically diverse perspective. Neural Regen Res. 2016;11(3):357-362.

58. Hartung D, Schafers M, Fujimoto S, et al. Targeting of matrix metalloproteinase activation for noninvasive detection of vulnerable atherosclerotic lesions. Eur J Nucl Med Mol Imaging. 2007;34(suppl 1): S1-S8.

59. Razavian M, Nie L, Challa A, et al. Lipid lowering and imaging protease activation in atherosclerosis. J Nucl Cardiol. 2014;21(2):319-328.

60. Schafers M, Riemann B, Kopka K, et al. Scintigraphic imaging of matrix metalloproteinase activity in the arterial wall in vivo. Circulation. 2004;109(21):2554-2559.

61. Mojtahedi A, Alavi A, Thamake S, et al. Assessment of vulnerable atherosclerotic and fibrotic plaques in coronary arteries using (68) Ga-DOTATATE PET/CT. Am J Nucl Med Mol Imaging. 2015;5(1): 65-71.

62. Michel JB, Martin-Ventura JL, Nicoletti A, Ho-Tin-Noe B. Pathology of human plaque vulnerability: mechanisms and consequences of intraplaque haemorrhages. Atherosclerosis. 2014;234(2):311-319.

63. Lu J, Duan W, Qiao A. Finite element analysis of mechanics of neovessels with intraplaque hemorrhage in carotid atherosclerosis. Biomed Eng Online. 2015;14(suppl 1):S3.

64. Humphrey JD. Vascular adaptation and mechanical homeostasis at tissue, cellular, and sub-cellular levels. Cell Biochem Biophys. 2008; 50(2):53-78.

65. Korshunov VA, Schwartz SM, Berk BC. Vascular remodeling: hemodynamic and biochemical mechanisms underlying Glagov's phenomenon. Arterioscler Thromb Vasc Biol. 2007;27(8):1722-1728.

66. Zhao X, Kong J, Zhao Y, et al. Gene silencing of TACE enhances plaque stability and improves vascular remodeling in a rabbit model of atherosclerosis. Sci Rep. 2015;5:17939.

67. Youn SW, Park KK. Small-nucleic-acid-based therapeutic strategy targeting the transcription factors regulating the vascular inflammation, remodeling and fibrosis in atherosclerosis. Int J Mol Sci. 2015;16(5): 11804-11833.

68. Kietselaer BL, Reutelingsperger CP, Heidendal GA, et al. Noninvasive detection of plaque instability with use of radiolabeled annexin A5 in patients with carotid-artery atherosclerosis. $N$ Engl J Med. 2004;350(14):1472-1473.

69. Zhao Y, Kuge Y, Zhao S, et al. Comparison of 99mTc-annexin A5 with $18 \mathrm{~F}-\mathrm{FDG}$ for the detection of atherosclerosis in ApoE-/- mice. Eur J Nucl Med Mol Imaging. 2007;34(11):1747-1755.

70. Zhao Y, Watanabe A, Zhao S, et al. Suppressive effects of irbesartan on inflammation and apoptosis in atherosclerotic plaques of ApoE-/-mice: molecular imaging with $14 \mathrm{C}-\mathrm{FDG}$ and $99 \mathrm{mTc}$-annexin A5. PLoS One. 2014;9(2):e89338. 
71. Isobe $\mathrm{S}$, Tsimikas $\mathrm{S}$, Zhou J, et al. Noninvasive imaging of atherosclerotic lesions in apolipoprotein E-deficient and low-density-lipoprotein receptor-deficient mice with annexin A5. J Nucl Med. 2006;47(9): $1497-1505$.

72. Tait JF, Smith C. Phosphatidylserine receptors: role of CD36 in binding of anionic phospholipid vesicles to monocytic cells. $J$ Biol Chem. 1999;274(5):3048-3054.

73. Henson PM, Bratton DL, Fadok VA. Apoptotic cell removal. Curr Biol. 2001;11(19):R795-R805.

74. Maiseyeu A, Mihai G, Kampfrath T, et al. Gadolinium-containing phosphatidylserine liposomes for molecular imaging of atherosclerosis. J Lipid Res. 2009;50(11):2157-2163.

75. Hight MR, Cheung YY, Nickels ML, et al. A peptide-based positron emission tomography probe for in vivo detection of caspase activity in apoptotic cells. Clin Cancer Res. 2014;20(8):2126-2135.

76. Winter PM, Neubauer AM, Caruthers SD, et al. Endothelial alpha(v) beta3 integrin-targeted fumagillin nanoparticles inhibit angiogenesis in atherosclerosis. Arterioscler Thromb Vasc Biol. 2006;26(9): 2103-2109.

77. Phinikaridou A, Andia ME, Indermuehle A, et al. Vascular remodeling and plaque vulnerability in a rabbit model of atherosclerosis: comparison of delayed-enhancement MR imaging with an elastin-specific contrast agent and unenhanced black-blood MR imaging. Radiology. 2014;271(2):390-399.

78. Zhang J, Razavian M, Tavakoli S, et al. Molecular imaging of vascular endothelial growth factor receptors in graft arteriosclerosis. Arterioscler Thromb Vasc Biol. 2012;32(8):1849-1855.

79. Longmire M, Choyke PL, Kobayashi H. Dendrimer-based contrast agents for molecular imaging. Curr Top Med Chem. 2008;8(14):1180-1186.

80. Thorp-Greenwood FL, Coogan MP. Multimodal radio-(PET/SPECT) and fluorescence imaging agents based on metallo-radioisotopes: current applications and prospects for development of new agents. Dalton Trans. 2011;40(23):6129-6143.

81. Parveen S, Misra R, Sahoo SK. Nanoparticles: a boon to drug delivery, therapeutics, diagnostics and imaging. Nanomedicine. 2012;8(2):147-166.

82. Xing $\mathrm{H}, \mathrm{Bu} \mathrm{W}$, Zhang $\mathrm{S}$, et al. Multifunctional nanoprobes for upconversion fluorescence, MR and CT trimodal imaging. Biomaterials. 2012;33(4):1079-1089.

83. Cheng D, Li X, Zhang C, et al. Detection of vulnerable atherosclerosis plaques with a dual-modal single-photon-emission computed tomography/magnetic resonance imaging probe targeting apoptotic macrophages. ACS Appl Mater Interfaces. 2015;7(4):2847-2855.

84. Hegde AR, Rewatkar PV, Manikkath J, Tupally K, Parekh HS, Mutalik S. Peptide dendrimer-conjugates of ketoprofen: synthesis and ex vivo and in vivo evaluations of passive diffusion, sonophoresis and iontophoresis for skin delivery. Eur J Pharm Sci. 2017;102: 237-249.

85. Jatczak-Pawlik I, Gorzkiewicz M, Studzian M, et al. Sugar-modified poly(propylene imine) dendrimers stimulate the NF-kappaB pathway in a myeloid cell line. Pharm Res. 2017;34(1):136-147.

86. Yu M, Jie X, Xu L, et al. Recent advances in dendrimer research for cardiovascular diseases. Biomacromolecules. 2015;16(9):2588-2598.

87. Markowicz M, Szymanski P, Ciszewski M, Klys A, Mikiciuk-Olasik E. Evaluation of poly(amidoamine) dendrimers as potential carriers of iminodiacetic derivatives using solubility studies and 2D-NOESY NMR spectroscopy. J Biol Phys. 2012;38(4):637-656.

88. Kobayashi H, Brechbiel MW. Nano-sized MRI contrast agents with dendrimer cores. Adv Drug Deliv Rev. 2005;57(15):2271-2286.

89. Menjoge AR, Kannan RM, Tomalia DA. Dendrimer-based drug and imaging conjugates: design considerations for nanomedical applications. Drug Discov Today. 2010;15(5-6):171-185.

90. Longmire MR, Ogawa M, Choyke PL, Kobayashi H. Dendrimers as high relaxivity MR contrast agents. Wiley Interdiscip Rev Nanomed Nanobiotechnol. 2014;6(2):155-162.

91. Kojima C, Turkbey B, Ogawa M, et al. Dendrimer-based MRI contrast agents: the effects of PEGylation on relaxivity and pharmacokinetics. Nanomedicine. 2011;7(6):1001-1008.
92. Kuil J, Buckle T, Oldenburg J, et al. Hybrid peptide dendrimers for imaging of chemokine receptor 4 (CXCR4) expression. Mol Pharm. 2011;8(6):2444-2453

93. Zhang Y, Xu X, Wang L, et al. Dendrimer-folate-copper conjugates as bioprobes for synchrotron X-ray fluorescence imaging. Chem Commun. 2013;49(88):10388-10390.

94. Klemm PJ, Floyd WC 3rd, Andolina CM, Frechet JM, Raymond KN. Conjugation to biocompatible dendrimers increases lanthanide T2 relaxivity of hydroxypyridinone (HOPO) complexes for magnetic resonance imaging (MRI). Eur J Inorg Chem. 2012;2012(12): 2108-2114.

95. Denora N, Laquintana $\mathrm{V}$, Lopalco $\mathrm{A}$, et al. In vitro targeting and imaging the translocator protein TSPO 18-kDa through G(4)-PAMAM-FITC labeled dendrimer. J Control Release. 2013;172(3):1111-1125.

96. Kim Y, Kim SH, Tanyeri M, Katzenellenbogen JA, Schroeder CM. Dendrimer probes for enhanced photostability and localization in fluorescence imaging. Biophys J. 2013;104(7):1566-1575.

97. Peng C, Zheng L, Chen Q, et al. PEGylated dendrimer-entrapped gold nanoparticles for in vivo blood pool and tumor imaging by computed tomography. Biomaterials. 2012;33(4):1107-1119.

98. Liu H, Wang $\mathrm{H}, \mathrm{Xu} \mathrm{Y}$, et al. Lactobionic acid-modified dendrimerentrapped gold nanoparticles for targeted computed tomography imaging of human hepatocellular carcinoma. ACS Appl Mater Interfaces. 2014;6(9):6944-6953.

99. Liu H, Wang H, Xu Y, et al. Synthesis of PEGylated low generation dendrimer-entrapped gold nanoparticles for CT imaging applications. Nanoscale. 2014;6(9):4521-4526.

100. Li K, Zhang Z, Zheng L, et al. Arg-Gly-Asp-D-Phe-Lys peptidemodified PEGylated dendrimer-entrapped gold nanoparticles for targeted computed tomography imaging of breast carcinoma. Nanomedicine (Lond). 2015;10(14):2185-2197.

101. Cai H, Li K, Li J, et al. Dendrimer-assisted formation of Fe3O4/Au nanocomposite particles for targeted dual mode CT/MR imaging of tumors. Small. 2015;11(35):4584-4593.

102. Chen Q, Wang H, Liu H, et al. Multifunctional dendrimer-entrapped gold nanoparticles modified with RGD peptide for targeted computed tomography/magnetic resonance dual-modal imaging of tumors. Anal Chem. 2015;87(7):3949-3956.

103. Li K, Wen S, Larson AC, et al. Multifunctional dendrimer-based nanoparticles for in vivo MR/CT dual-modal molecular imaging of breast cancer. Int J Nanomedicine. 2013;8:2589-2600.

104. Chen Q, Li K, Wen S, et al. Targeted CT/MR dual mode imaging of tumors using multifunctional dendrimer-entrapped gold nanoparticles. Biomaterials. 2013;34(21):5200-5209.

105. Abboud D, Hanson J. Chemokine neutralization as an innovative therapeutic strategy for atopic dermatitis. Drug Discov Today. 2017; 22(4):702-711.

106. Luong D, Sau S, Kesharwani P, Iyer AK. Polyvalent folate-dendrimercoated iron oxide theranostic nanoparticles for simultaneous magnetic resonance imaging and precise cancer cell targeting. Biomacromolecules. 2017;18(4):1197-1209.

107. Ye K, Qin J, Peng Z, et al. Polyethylene glycol-modified dendrimerentrapped gold nanoparticles enhance CT imaging of blood pool in atherosclerotic mice. Nanoscale Res Lett. 2014;9(1):529.

108. Qin J, Peng C, Zhao B, et al. Noninvasive detection of macrophages in atherosclerotic lesions by computed tomography enhanced with PEGylated gold nanoparticles. Int J Nanomedicine. 2014;9: 5575-5590.

109. Seo JW, Baek H, Mahakian LM, et al. (64)Cu-labeled LyP-1dendrimer for PET-CT imaging of atherosclerotic plaque. Bioconjug Chem. 2014;25(2):231-239.

110. Nguyen TH, Bryant H, Shapsa A, et al. Manganese G8 dendrimers targeted to oxidation-specific epitopes: in vivo MR imaging of atherosclerosis. J Magn Reson Imaging. 2015;41(3):797-805.

111. Choudhary S, Gupta L, Rani S, Dave K, Gupta U. Impact of dendrimers on solubility of hydrophobic drug molecules. Front Pharmacol. 2017;8:261. 
112. Wilczewska AZ, Niemirowicz K, Markiewicz KH, Car H. Nanoparticles as drug delivery systems. Pharmacol Rep. 2012;64(5):1020-1037.

113. Bahadir EB, Sezginturk MK. Poly(amidoamine) (PAMAM): an emerging material for electrochemical bio(sensing) applications. Talanta. 2016;148:427-438.
114. Chan JMS, Monaco C, Wylezinska-Arridge M, et al. Imaging vulnerable plaques by targeting inflammation in atherosclerosis using fluorescent-labeled dual-ligand microparticles of iron oxide and magnetic resonance imaging. J Vasc Surg. Epub 2017 Jun 22.

\section{Publish your work in this journal}

The International Journal of Nanomedicine is an international, peerreviewed journal focusing on the application of nanotechnology in diagnostics, therapeutics, and drug delivery systems throughout the biomedical field. This journal is indexed on PubMed Central,

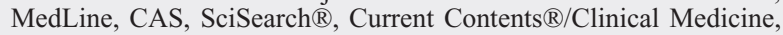

Journal Citation Reports/Science Edition, EMBase, Scopus and the Elsevier Bibliographic databases. The manuscript management system is completely online and includes a very quick and fair peer-review system, which is all easy to use. Visit http://www.dovepress.com/ testimonials.php to read real quotes from published authors.

Submit your manuscript here: http://www.dovepress.com/international-journal-of-nanomedicine-journal 